\title{
KALMAN FILTERING OF EARTH ORIENTATION CHANGES
}

\author{
David D. Morabito, T. Marshall Eubanks, and J. Alan Steppe \\ Jet Propulsion Laboratory \\ California Institute of Technology \\ Pasadena, California 91109
}

\begin{abstract}
The orientation of the earth in space changes unpredictably in a rapid and irregular manner, in addition to the uniform rotation of the earth. Observations of extra-terrestrial objects from the surface of the earth are affected by these variations, and knowledge of these changes is required for a variety of geodetic and astrometric purposes as well as being of interest in its own right. The orientation of the earth (specified by a three dimensional rotation vector) is measured by a variety of techniques; combination of these data sets is complicated by irregular changes in the spacing and accuracy of the various time series, and also by the existence of lower dimensional measurements of different linear combinations of the rotation vector components. A Kalman filter has been developed at JPL to smooth and predict earth orientation changes for application to spacecraft navigation by the NASA Deep Space Network. The filter, which provides estimates of the earth orientation changes (and of the excitation of these changes) based on whatever measurements are available, has been used for a number of research applications, both in the reduction of geodetic and astrometric data, and in research into the geophysical causes of earth orientation changes. The JPL Kalman filter uses stochastic models to account statistically for otherwise unpredictable changes in earth orientation; these models make it possible to provide reasonable estimates of the error in the smoothed time series, and to automatically vary the amount of smoothing according to the accuracy and density of the data. The derivation of the stochastic models used by the filter, the implementation of the models into the filter, a statistical description of what the filter does, and the results of filtering specific data sets will be discussed.
\end{abstract}

\section{INTRODUCTION}

The earth does not rotate as a rigid body but undergoes very small variations in rotation (UT1) and in the position of the pole (polar motion). There are unpredictable rapid oscillations in these quantities which are primarily driven by the exchange of angular momentum between the atmosphere and the "solid" earth (the crust and mantle) and by deformations of the "solid" earth. Such changes are continuously monitored through observations of extraterrestrial objects from the surface of the earth using a variety of space-age techniques. These techniques include very long baseline interferometry (VLBI), satellite laser ranging (SLR), and lunar laser ranging (LLR). Two techniques, VLBI and SLR, monitor the polar motion on a regular basis with a demonstrated accuracy of $\approx 2$ mas (milliarcseconds) in each component (one mas $\approx 3.1 \mathrm{~cm}$ at the surface of the earth), while VLBI and LLR are capable of similar accuracy in measurements of UT1. Such measurements are a factor of 5 to 10 times more accurate than those from older techniques (such as optical astrometry), and have enabled detection of much smaller motions than was previously possible. Regular independent VLBI estimates of the earth orientation are 
routinely produced by POLARIS/IRIS (Carter and Robertson, 1984) and by the TEMPO project of the NASA Deep Space Network at JPL (Eubanks et al. 1982).

There are several problems which exist in the processing of these earth rotation measurements; 1) there are no deterministic models available to describe the rapid oscillations in the earth orientation, 2) the data from the different measurement techniques are of irregular quality and have a wide range of formal errors, 3) the data are taken at irregular intervals, and 4) the measurements are sometimes of lower rank. Kalman filtering and auto-regressive time series analysis have been combined in order to address these problems. The Kalman filter offers a number of advantages in filtering and smoothing multiple dimensional time series such as UT1 and polar motion. It was designed to provide the NASA Deep Space Network with accurate estimates of earth orientation changes during periods of spacecraft navigation. With this filter, it is possible to produce both predictions and smoothings using raw measurements at irregular times with widely varying accuracies. The filter can be used for a variety of applications; 1 ) interpolation and prediction of earth orientation changes for application to spacecraft navigation, 2) intercomparision of orientation measurements from different techniques, 3) removal of earth orientation effects from estimates of regional and global plate motions, and 4) analysis of the excitations of the polar motion for various research purposes. Stochastic models have been derived for the UT1 and polar motion changes, primarily from analysis of atmospheric angular momentum (AAM) data (Eubanks et al. 1985). The derivation of the stochastic model, its implementation into the Kalman filter, a statistical description of the filter, and intercomparisions between various data sets using the filter will be discussed.

\section{STOCHASTIC MODELS FOR THE KALMAN FILTER}

The Kalman filter is designed for sequential estimation when a stochastic model exists and when the data contains noise. For general discussions on Kalman filtering, the reader is referred to the references (Bierman; Gelb; Nahi). The geophysical signals are all "red noises" i.e. their power spectra decline with increasing frequency. Since the measurement noise is assumed to be white, the filter, which attenuates regions of the spectrum where the signal-to-noise ratio is small, is in practice a time-variable multi-dimensional low pass filter.

The input to the filter consists of raw UT1 and polar motion measurements, and the measurement formal error covariance matrices, after removal of a model of the short period tidal variations to the UT1 (Yoder, Williams and Parke, 1981). These tidal variations were not directly modeled in the filter since they can be adequately modeled theoretically and are at high enough frequencies to be partially attenuated by the filter. The Barnes et al. (1983) formulation is used in the modeling of the excitations and the earth orientation quantities. The output of the filter consists of estimates of UT1 and polar motion, estimates of the excitation vector of the earth orientation parameters, and estimates of the covariance matrix.

The power spectra of the excitation functions largely control the design of the filter. In filter design, the most important region of the spectrum to model accurately is the region where the geophysical signal-to-noise power ratio is near unity, but this is generally difficult to model since the signal is obscured by noise. The AAM data provide a means of extrapolating the observed low frequency geodetic spectrum to the higher frequencies dominated by geodetic measurement noise. The agreement between the raw and smoothed data has been used to establish the adequacy of the model and to "fine-tune" the strength of the stochastic forcing. To simplify filter implementation, the total stochastic model was chosen to be a sum of simple stochastic processes.

The polar motion is dominated by the Chandler wobble, the resonant oscillation predicted by Euler refined to include the elasticity of the earth. This oscillation involves deviations of about 200 mas of the rotation axis about the axis of greatest moment of inertia. The observed polar 
motion is the response of the Chandler wobble resonance to the excitations, denoted by $\chi_{1}$ and $\chi_{2}$, and is described as follows;

$$
\frac{d}{d t}\left(\begin{array}{l}
P M X \\
P M Y
\end{array}\right)=\sigma\left(\begin{array}{cc}
-\frac{1}{2 Q} & 1 \\
-1 & -\frac{1}{2 Q}
\end{array}\right)\left(\begin{array}{c}
P M X \\
P M Y
\end{array}\right)+\sigma\left(\begin{array}{cc}
\frac{1}{2 Q} & 1 \\
1 & -\frac{1}{2 Q}
\end{array}\right)\left(\begin{array}{l}
\chi_{1} \\
\chi_{2}
\end{array}\right)
$$

where $Q$ is the polar motion quality factor, taken to be 170 , and $\sigma$ is the Chandler wobble frequency $\left(2 \pi / 433\right.$ day $\left.^{-1}\right) . \chi_{1}$ and $\chi_{2}$ are themselves also modeled as stochastic processes, the combination of a random walk $\mu$ and a seasonal process $S$.

The seasonal variations in $\chi_{1}$ and $\chi_{2}$ are best described by study of long time series of polar motion from the International Latitude Service (ILS). Such studies reveal that the annual variation is nearly linearly polarized and that the major variation is fortuitously along the $\chi_{2}$ axis. The $\chi_{2}$ excitation component model contains an auto-regressive process which characterizes the annual wobble. It was decided to model this variation explicitly by the addition of a second-order auto-regressive process, $S$, which is essentially a resonant oscillator driven by noise. $S$ is described by the equation $\ddot{S}+\alpha_{1} \dot{S}+\alpha_{2} S=\omega_{S}$, which can be rewritten;

$$
\frac{d}{d t}\left(\begin{array}{c}
S \\
\dot{S}
\end{array}\right)=\left(\begin{array}{cc}
0 & 1 \\
-\alpha_{2} & -\alpha_{1}
\end{array}\right)\left(\begin{array}{l}
S \\
\dot{S}
\end{array}\right)+\left(\begin{array}{c}
0 \\
\omega_{S}
\end{array}\right)
$$

$\alpha_{1}$ and $\alpha_{2}$ are coefficients describing the auto-regressive process where the dissapation time is given by $\frac{2}{\alpha_{1}}$ and the resonance period is given by $2 \pi\left(\alpha_{2}-\frac{\alpha_{1}^{2}}{4}\right)^{-1 / 2}$. The coefficients $\alpha_{1}$ $=.002 \mathrm{day}^{-1}$ and $\alpha_{2}=2.9692338 * 10^{-4} \mathrm{day}^{-2}$ were chosen to give a resonance period of one year and a damping time of 1000 days. The white noise, $\omega_{S}$, was chosen to have a power spectral density $Q_{S}=4.26 * 10^{-4} \mathrm{mas}^{2} / \mathrm{day}^{3}$. The strength of the white noise function implies a steady state seasonal $\chi_{2}$ variation of $\approx 42$ mas amplitude.

The model for the two components of polar motion excitation also contains an isotropic random walk with equal noise $\left(\omega_{\mu_{1}}\right.$ and $\left.\omega_{\mu_{2}}\right)$ forcing both components and having a white noise power spectral density $Q_{\mu}$ of $246.6 \mathrm{mas}^{2} /$ day. The full model for the polar motion excitations is thus $\chi_{1}=\mu_{1}$ and $\chi_{2}=\mu_{2}+S$, where $\mu_{i}$ denotes the random walk process on $\chi_{i}$.

Figure 1 displays the spectra of the meteorological excitation estimates for $\chi_{1}$ and $\chi_{2}$ along with the spectrum of the random walk model flanked by its 99 percent confidence limits. The spectra were derived from pressure term AAM data. In order for the filter to work properly, the stochastic model must be appropriate in the region of the spectrum where the signal-to-noise ratio lies near unity, which for this case is the region with periods of 20 to 40 days. As a result of this spectral analysis, a stochastic model for the two components of excitation to the polar motion has been chosen. A more complex model may be implemented in the future whose power spectra more closely fit the observed excitation spectra.

The UT1 filter requires a statistical model to describe the unpredictable high frequency UT1 fluctuations. Conservation of angular momentum implies that the variation in length of day $(L O D)$ is equal, aside from a change of units, to $\chi_{3}$, the polar component of AAM (Eubanks et al. 1985). The power spectrum of the $\chi_{3}$ derived from the AAM wind data is displayed in figure 2 along with the $f^{-2}$ power law of the random walk model flanked by its 99 percent confidence limits. The strong annual and semiannual peaks visible in the raw data can be removed by subtraction of best-fit sinusoids resulting in the seasonally adjusted power spectrum. The seasonally adjusted spectrum fits the random walk model very well at virtually all frequencies. The UT1 can thus be modeled as an integrated random walk described by the following equation;

$$
-\frac{d^{2}}{d t^{2}} U T 1 R=\frac{1}{L O D_{0}} \frac{d}{d t} L O D R=\omega_{L}
$$

where UT1R is the earth rotation universal time with tides removed, LODR is the variation of the length of day with tides removed, $L O D_{0}=86400 \mathrm{sec}$, and $\omega_{L}$ is a white noise process. The 


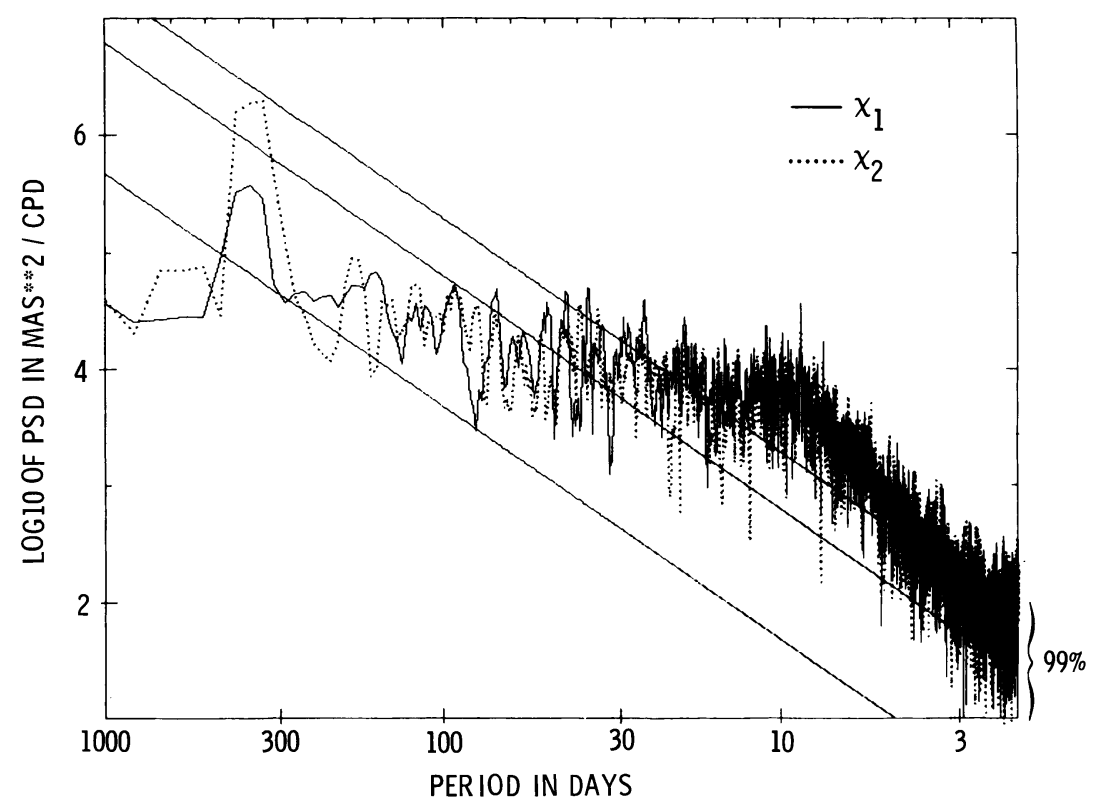

Figure 1. Power Spectra of $x_{1}$ and $x_{2}$ Derived from AAM Data (Pressure Term) with Superimposed Rândom Wa1k Mode1.

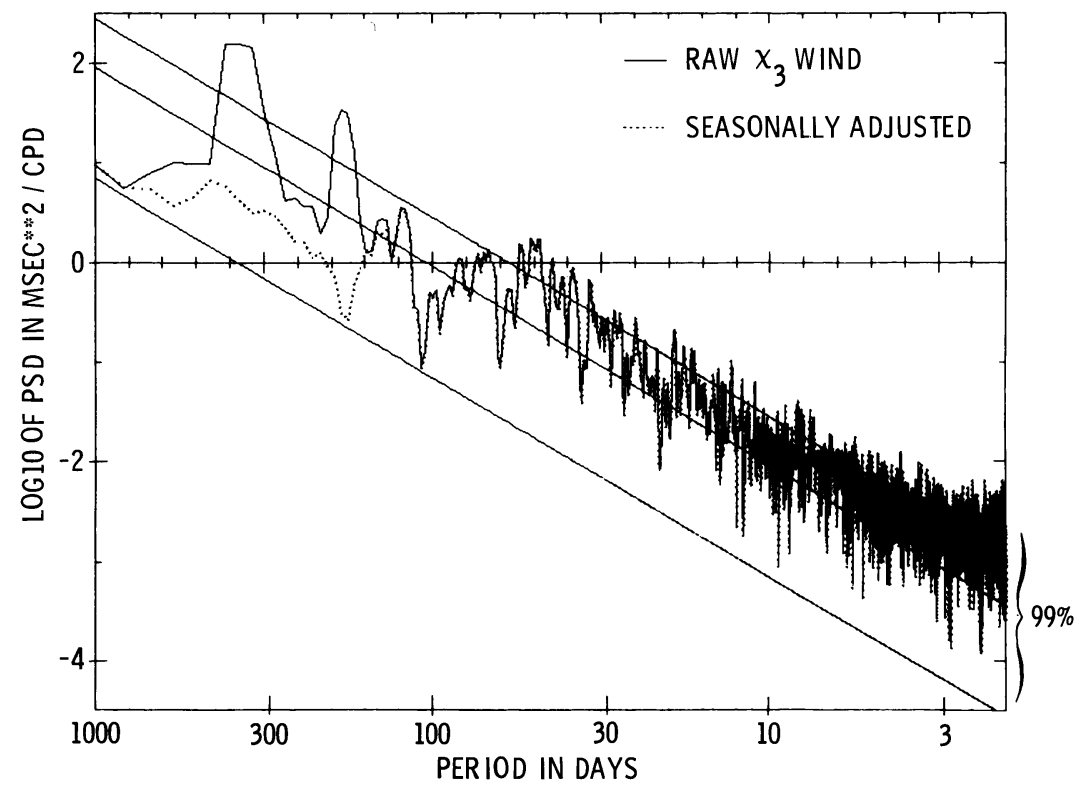

Figure 2. Power Spectrum of $x_{3}$ Derived from AAM Data (Wind Term) with Superimposed Random Wa1k Mode1. 
excitation to the UT1 is modeled as a random walk forced by white noise of power spectral density $Q_{L}=0.0036 \mathrm{msec}^{2} / \mathrm{day}^{3}$. The resulting system of equations is given by;

$$
\frac{d}{d t}\left(\begin{array}{c}
U T 1 R \\
L O D R_{r}
\end{array}\right)=\left(\begin{array}{rr}
0 & -1 \\
0 & 0
\end{array}\right)\left(\begin{array}{c}
U T 1 R \\
L O D R_{r}
\end{array}\right)+\left(\begin{array}{c}
0 \\
\omega_{L}
\end{array}\right)
$$

where $L O D R_{r}=L O D R / L O D_{0}$.

\section{KALMAN FILTER FOR UT1 AND POLAR MOTION}

Given a linear stochastic model, it is easy to derive the corresponding optimal Kalman filter. We have a model vector process $X$, expressed by a linear first order stochastic differential equation driven by a zero mean white noise vector, $\omega$, with constant coefficient matrices $F$ and $G$;

$$
\frac{d X}{d t}=F X+G \omega
$$

where

$$
X=\left(\begin{array}{c}
P M X \\
P M Y \\
\mu_{1} \\
\mu_{2} \\
S \\
\dot{S} \\
U T 1 R \\
L O D R_{r}
\end{array}\right) \quad \omega=\left(\begin{array}{c}
0 \\
0 \\
\omega_{\mu_{1}} \\
\omega_{\mu_{2}} \\
0 \\
\omega_{S} \\
0 \\
\omega_{L}
\end{array}\right)
$$

$X$ is the state vector where the entries have been previously defined.

The dynamics of the system described by equations 1-3 are incorporated into the constant coefficient matrices $F$ and $G$ given by;

$$
\begin{aligned}
& F=\left(\begin{array}{cccccccr}
-\sigma / 2 Q & \sigma & \sigma / 2 Q & \sigma & \sigma & 0 & 0 & 0 \\
-\sigma & -\sigma / 2 Q & \sigma & -\sigma / 2 Q & -\sigma / 2 Q & 0 & 0 & 0 \\
0 & 0 & 0 & 0 & 0 & 0 & 0 & 0 \\
0 & 0 & 0 & 0 & 0 & 0 & 0 & 0 \\
0 & 0 & 0 & 0 & 0 & 1 & 0 & 0 \\
0 & 0 & 0 & 0 & -\alpha_{2} & -\alpha_{1} & 0 & 0 \\
0 & 0 & 0 & 0 & 0 & 0 & 0 & -1 \\
0 & 0 & 0 & 0 & 0 & 0 & 0 & 0
\end{array}\right) \\
& G=\left(\begin{array}{llllllll}
0 & 0 & 0 & 0 & 0 & 0 & 0 & 0 \\
0 & 0 & 0 & 0 & 0 & 0 & 0 & 0 \\
0 & 0 & 1 & 0 & 0 & 0 & 0 & 0 \\
0 & 0 & 0 & 1 & 0 & 0 & 0 & 0 \\
0 & 0 & 0 & 0 & 0 & 0 & 0 & 0 \\
0 & 0 & 0 & 0 & 0 & 1 & 0 & 0 \\
0 & 0 & 0 & 0 & 0 & 0 & 0 & 0 \\
0 & 0 & 0 & 0 & 0 & 0 & 0 & 1
\end{array}\right)
\end{aligned}
$$

A solution for $X(t)$ is:

$$
X(t)=\phi\left(t-t_{0}\right) X\left(t_{0}\right)+\int_{t_{0}}^{t} \phi(t-\tau) G \omega(\tau) d \tau
$$


where $X\left(t_{0}\right)$ is an initial state, and $\phi(t)$, which is called the transition matrix, can be expressed as an exponential series in terms of $F$ and $t$;

$$
\phi(t)=e^{F t}=\sum_{k=0}^{\infty} \frac{F^{k} t^{k}}{k !}
$$

The actual calculation of $\phi(t)$ involves partitioning the $F$ matrix into submatrices and then deriving a combination of analytic and computational solutions. The known part or solution in the absence of noise is given by the first term on the right hand side of equation 4 , and the unknown part is given by the second term. The unknown part, an integral of the white noise, describes the unpredictable rapid fluctuations in the earth rotation.

Now suppose an initial estimate $\hat{X}(t)$ plus a measurement $Z(t)$ are available. The initial estimate $\hat{X}(t)$ is equal to the quantity we are trying to measure, $X(t)$, corrupted by filter noise whose covariance matrix is given by $P_{t}$. The measurement $Z(t)$ is equal to the quantity we are trying to measure, $X(t)$, corrupted by measurement noise whose inverse covariance matrix is given by $R_{t}$. When a new measurement is available, the optimum state estimate consists of the vector weighted average of the propagated state and the new measurement:

$$
\hat{X}(t)^{+}=\left(P_{t}^{-1}+R_{t}\right)^{-1}\left[P_{t}^{-1} \hat{X}(t)+R_{t} Z(t)\right]
$$

The filter is recursive, that is each new estimate is computed from the previous estimates plus any new incoming data. Given an initial estimate, $\hat{X}\left(t_{i}\right)$, and a covariance matrix, $P_{t_{i}}$, the filter is used to propagate to the time of the next measurement $t_{i+1}$. The new estimate and its covariance matrix are given by:

$$
\begin{gathered}
\hat{X}\left(t_{i+1}\right)=\phi\left(t_{i+1}-t_{i}\right) \hat{X}\left(t_{i}\right)^{+} \\
P_{t_{i+1}}=\phi P_{t_{i}} \phi^{T}+\int_{t_{i}}^{t_{i+1}} \phi\left(t_{i+1}-\tau\right) G \tilde{Q} G^{T} \phi^{T}\left(t_{i+1}-\tau\right) d \tau
\end{gathered}
$$

The process noise matrix $\tilde{Q}$ is given by;

$$
\tilde{Q}=\left(\begin{array}{cccccccc}
0 & 0 & 0 & 0 & 0 & 0 & 0 & 0 \\
0 & 0 & 0 & 0 & 0 & 0 & 0 & 0 \\
0 & 0 & Q_{\mu} & 0 & 0 & 0 & 0 & 0 \\
0 & 0 & 0 & Q_{\mu} & 0 & 0 & 0 & 0 \\
0 & 0 & 0 & 0 & 0 & 0 & 0 & 0 \\
0 & 0 & 0 & 0 & 0 & Q_{S} & 0 & 0 \\
0 & 0 & 0 & 0 & 0 & 0 & 0 & 0 \\
0 & 0 & 0 & 0 & 0 & 0 & 0 & Q_{L}
\end{array}\right)
$$

The new covariance matrix (equation 5 ) is given by two terms, the first being due to the initial error at time $t_{i}$ and the second being due to the effect of unmodeled excitation (process noise). The error in orientation grows without new measurements due to both initial errors associated with the apriori estimate and unknown perturbations which can't be accounted for without new measurements. If the state is known at some initial time, then the additional error introduced after $d$ days (for $d \ll 365$ days) without additional measurements is $\sigma=K d^{3 / 2}$ where $K$ is 0.52 and $0.13 \mathrm{mas} / \mathrm{day}^{3 / 2}$ for UT1 and polar motion respectively. The UT1 error grows four times as fast as the polar motion error. In practice, of course, the initial conditions are never known exactly, and the total prediction error is approximately the rss of the above error and the propagated effect of any initial errors, which grow linearly with time. Although the Kalman filter 


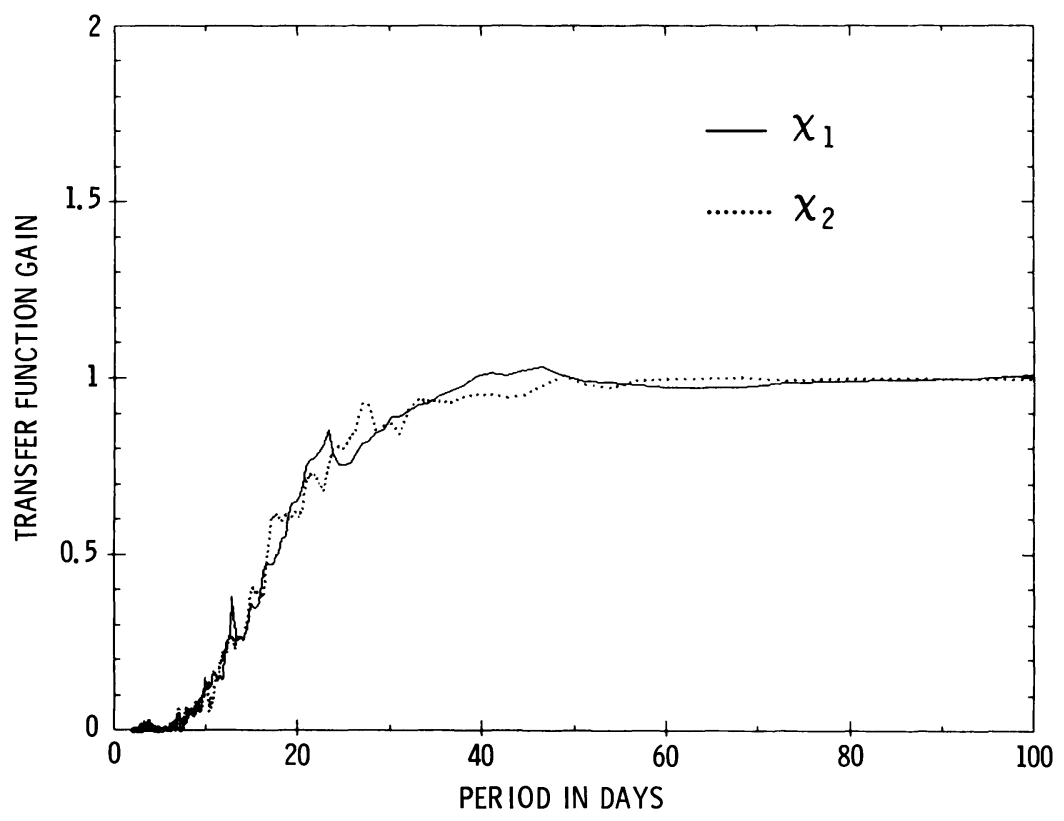

Figure 3. Raw $\longrightarrow$ Smoothed Transfer Function Gain of Kalman Smoother.

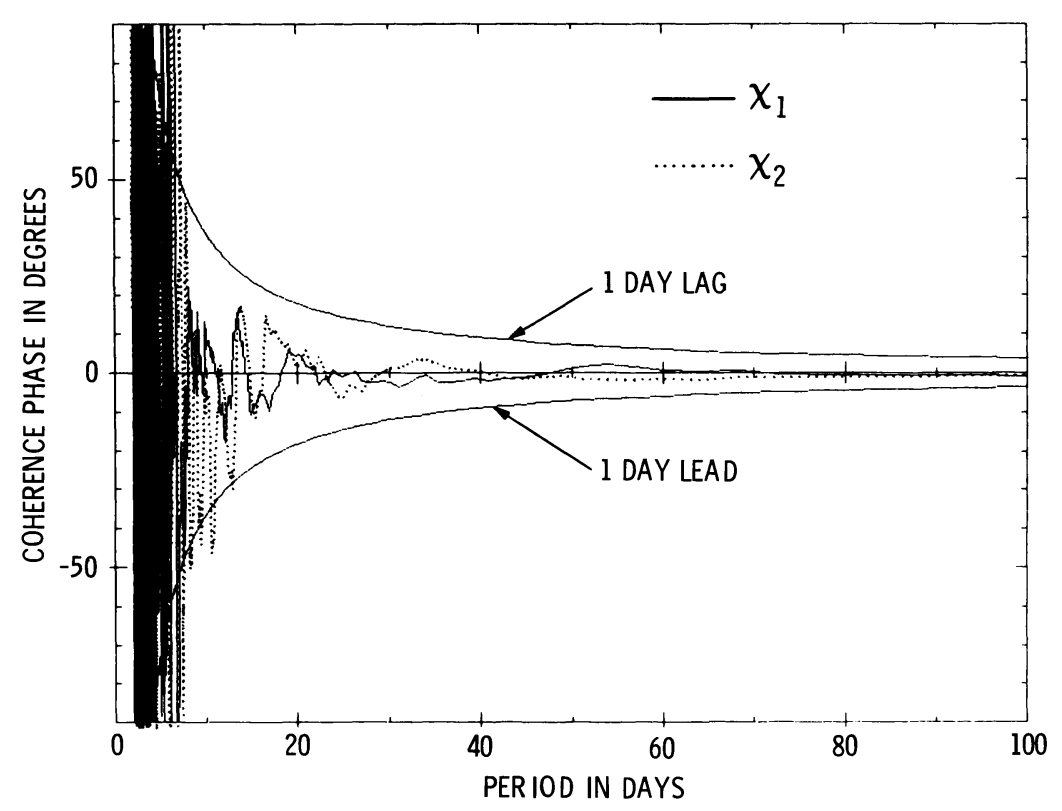

Figure 4. Raw $\longrightarrow$ Smoothed Transfer Function Phase of Kalman Smoother. 
calculates the measurement errors rigorously, this approximation has proved to be very useful for mission planning and error budget calculations.

The filter uses only data up to and including the time of the estimate. The smoother uses all of the data to produce the estimate. The backward filter (which is equivalent to the forward filter substituting $-\mathrm{T}$ for $\mathrm{T}$ into the equations) and the forward filter produce independent estimates. The optimal smoother is a weighted vector average of the estimates of the forward and backward filters.

\section{STATISTICAL DESCRIPTION OF THE FILTER}

A set of meteorologically derived excitations for 1984 to 1985 was convolved into the corresponding earth orientation changes by numerical integration of equation 1 . The resulting data set was then treated as if it were a real geodetic data set and was assigned measurement inverse covariance matrices (or $R_{t}$ ) from a real geodetic data set. The data were then input into the Kalman smoother and the output excitation estimates were compared with the raw meteorological excitations. The transfer function of the filter was computed from the raw and smoothed excitation series as described in Eubanks et al. (1985). The gain of this transfer function, shown in figure 3, represents the real number that when multiplied by the amplitude of a particular sinusoid in the raw data, produces the amplitude of the corresponding sinusoid in the smoothed data. It is evident from figure 3 that there is unity gain for components with periods of greater than about 40 days (low frequencies), and that the filter effectively blocks out components with periods of less than 5 days (high frequencies). The Kalman filter thus behaves as a low pass filter. Figure 4 displays the transfer function (coherence) phase between the raw and smoothed data for both components of the excitation. This is the phase in degrees between two corresponding sinusoids in the raw and the smoothed data. For periods of less than 5 days, where there is essentially no signal passed by the filter, the phase is degraded by quantization error. Note that the phase between the raw and smoothed data is less than the one day spacing of the data essentially everywhere. The analysis of the filter transfer function of the excitation for the UT1 produces similar results.

\section{INTERCOMPARISIONS OF DATA TYPES}

The adequacy of the filter models can be tested from comparison of raw and smoothed geodetic data in the time domain. Displayed in figure 5 are both components of polar motion from the raw IRIS multi-baseline VLBI, along with the Kalman smoothed IRIS VLBI polar motion and the Kalman smoothed SLR polar motion, all with respect to BIH Circular D. The smoothings between different data sets agree closely demonstrating the ability of the filter to track higher frequency variations than the BIH Circular D is sensitive to. Displayed in figure 6 are the raw IRIS multibaseline UT1R, the Kalman smoothed IRIS UT1R (solid line) and the Kalman smoothed DSN VLBI plus LLR UT1R (dotted line), all with respect to BIH Circular D. It is evident from this figure that the smoothing of the IRIS data agrees well with the raw IRIS data, and that the independent smoothing of the combined DSN VLBI and LLR data sets tracks the variations seen by the IRIS VLBI smoothing reasonably well.

\section{CONCLUSION}

A stochastic model for earth rotation has been derived using atmospheric angular momentum data, and a Kalman filter has been developed based on this model. A statistical description of the filter has been provided, along with demonstrations of the performance of the filter using data from different measurement techniques. 


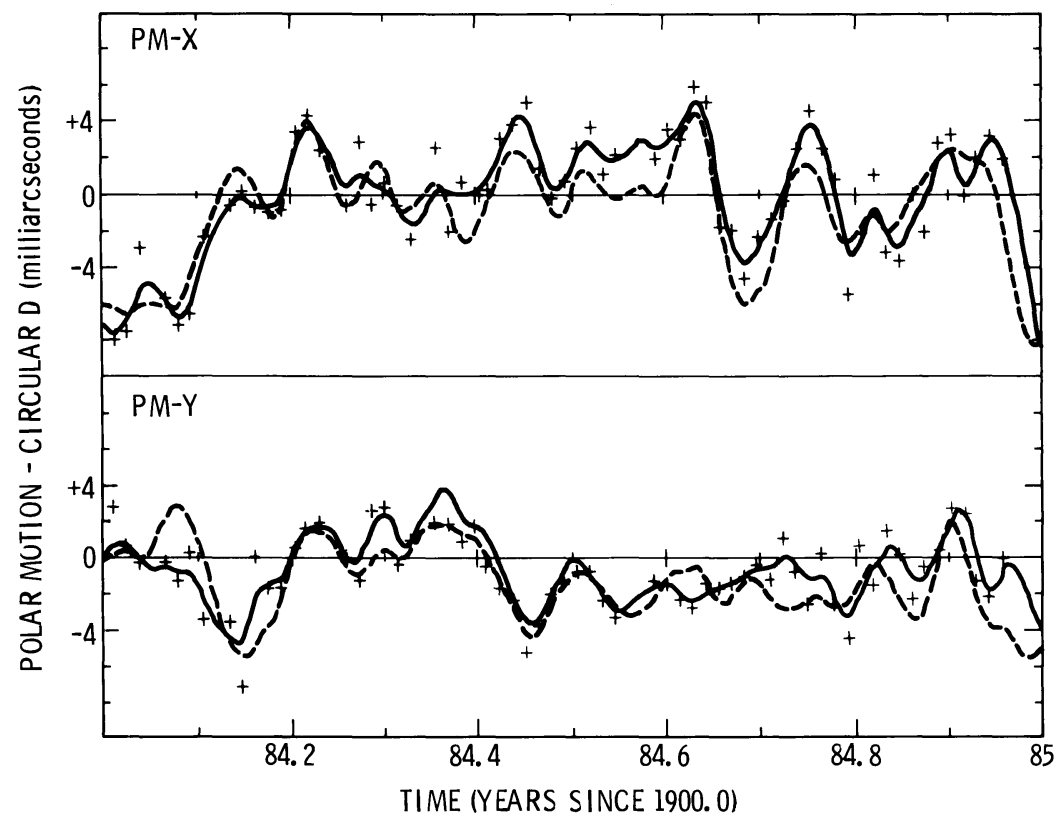

Figure 5. Polar Motion Intercomparison of Raw IRIS, Kalman Smoothed IRIS (Solid Line), Kalman Smoothed SLR (Dotted Line).

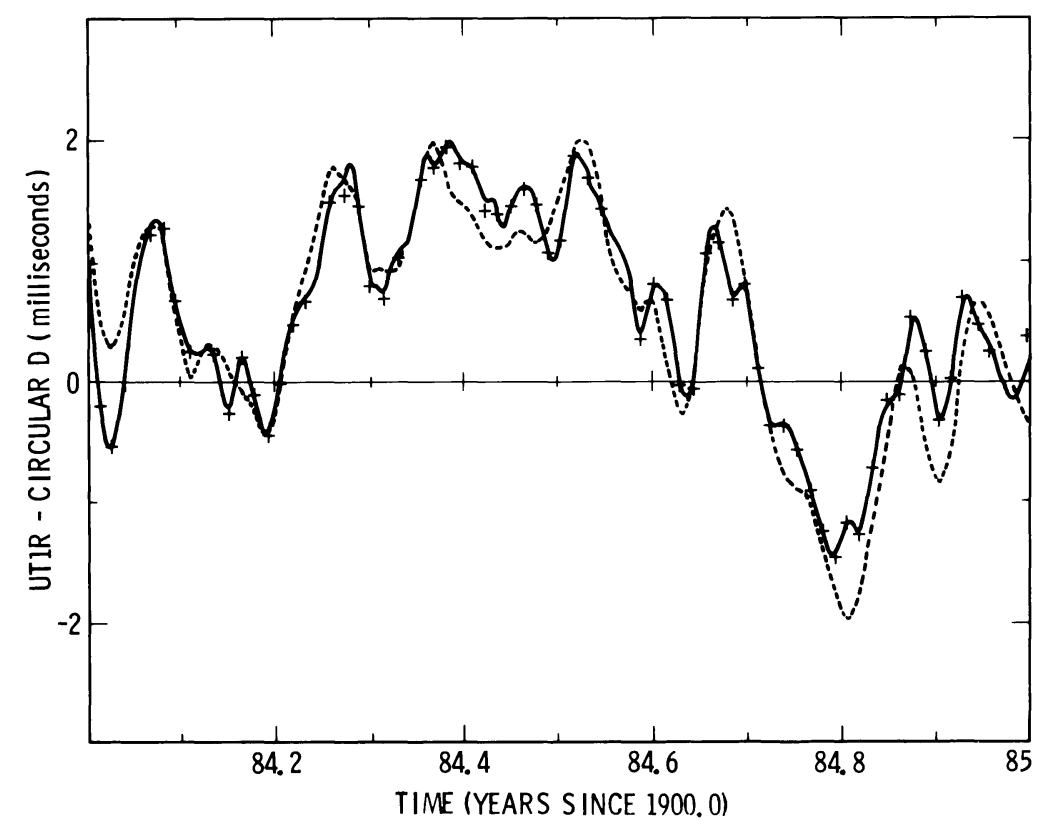

Figure 6. UT1 Intercomparison of Raw IRIS, KaLman Smoothed IRIS (Solid Line), Kalman Smoothed DSN VLBI Plus LLR (Dotted Line). 
ACKNOWLEDGMENTS. The authors would like to acknowledge the contributions of D. Hernquist in the development of the filter. This work was supported by the Jet Propulsion Laboratory, California Institute of Technology, under contract with the National Aeronautics and Space Administration.

\section{REFERENCES}

Barnes, R.T.H., Hide, R., White, A.A., and Wilson, C.A., "Atmospheric Angular Momentum Fluctuations Correlated with Length of Day Changes and Polar Motion", Proc. R. Soc. Lond. Ser. A, 387, 31-73, (1983).

Bierman, G.J., Factorization Methods for Discrete Sequential Estimation, Academic Press, New York 1977.

Carter, W.E., and Robertson, D.S., "IRIS Earth Rotation and Polar Motion Results", in Proc. Int. Symp. Space techniques for Geodynamics, (Sopron, Hungary), 1984.

Eubanks, T.M., Roth, M.G., Esposito, P.B., Steppe, J.A., and Callahan, P.S., "An Analysis of JPL TEMPO Earth Orientation Results", Proceedings of Symposium 5; Geodetic Applications of Radio Interferometry, NOAA Tech. Rep., 95, NGS 24, 81-90, 1982.

Eubanks, T.M., Steppe, J.A., Dickey J.O., and Callahan, P.S., "A Spectral Analysis of the Earth's Angular Momentum Budget”, Journal of Geophysical Research, 90, pp. 5385-5404. June 10, 1985.

Gelb, A., editor Applied Optimal Estimation, Cambridge, MA: MIT Press, 1974.

Nahi, N.E., Estimation Theory and Applications, John Wiley and Sons, New York, 1969.

Yoder, C.F., Williams, J.G., and Parke, M.E., "Tidal Variations of Earth Rotation", Journal of Geophysical Research, 86, 881-891, 1981. 


\section{DISCUSSION}

Feissel: I would like to make two comments.

- Some combination algorithms include smoothing of time series as an intermediate step. The characteristics of the smoothing usually reflect a trade-off between the a priori estimation of the measurement noise spectrum and the Earth's rotation noise spectrum. Therefore they influence the noise spectrum of the combined solution. To avoid this drawback, the organisation of the combined solution in a series of independent normal points at regular time intervals is preferable. Smoothing can then be performed if necessary, as post-processing. The normal values provide an appropriate material for scientific investigations.

- The key for consistency of the combined series is the monitoring of the reference frames, terrestrial and celestial, to which the individual series are referred, and of relative displacements. Indeed, the combination algorithm must then also make provisions for the effects of any modelling errors in each of the observing techniques.

McCarthy: 1) We have heard that the importance of Atmospheric Angular Momentum (AAM) in the determination of polar motion is not proven. Why do you go to such lengths to incorporate this in your solution? 2) What is the weight of the AAM contribution to the combination?

Reply by Morabito: 1) The AAM data were used only in the derivation of the stochastic models used by the filter. The AAM data provide a means of extrapolating the observed low frequency geodetic spectrum to the higher frequencies dominated by geodetic measurement noise. The adequacy of the model has been established upon examination of the agreement between the raw and smoothed data. Recent work by Eubanks, Steppe and Dickey show that observed rapid polar motions are correlated with atmospheric pressure changes. 2) The AAM data are not used as an input data type to the filter. They were used only in the derivation of the stochastic models used by the filter.

Herring: Why is the 2nd order autoregressive process added to $\chi_{2}$ ?

Reply by Morabito: Our study of the atmospheric excitation data shows a peak in the spectrum for $\chi_{2}$ at the annual period. Studies of seasonal variations in $\chi_{1}$ and $\chi_{2}$ using International Latitude Service data reveal that the annual variation is nearly linearly polarized and that the major component lies fortuitously along the $\chi_{2}$ axis. It was decided to model this variation by the addition of a second order auto-regressive process to the model for $\chi_{2}$. 